\title{
Classical and Quasi-Classical Consideration of Charged Particles in Coulomb Field of Bound Charges
}

\author{
Stanislav Podosenov ${ }^{1}$, Jaykov Foukzon ${ }^{2}$, Alexander Potapov ${ }^{3}$, and Elena \\ Men'kova ${ }^{4}$ \\ ${ }^{1}$ All-Russian Research Institute of Optophysical Measurements, Moscow, Russia \\ ${ }^{2}$ Israel Institute of Technology, Haifa, Israel \\ ${ }^{3}$ Kotel'nikov Institute of Radioengineering and Electronics of the Russian Academy of \\ Sciences, Moscow, Russia \\ ${ }^{4}$ All-Russian Research Institute of Optophysical Measurements, Moscow, Russia
}

\begin{abstract}
On the basis of the theory of bound charges the calculation of the motion of the charged particle at the Coulomb field formed with the spherical source of bound charges is carried out. Such motion is possible in the Riemanniam space-time. The comparison with the general relativity theory (GRT) and special relativity theory (SRT) results in the Schwarzshil'd field when the particle falls on the Schwarzshil'd and Coulomb centres is carried out. It is shown that the proton and electron can to create a stable connection with the dimensions of the order of the classic electron radius. The perihelion shift of the electron orbit in the proton field is calculated. This shift is five times greater than in SRT and when corrsponding substitution of the constants it is 5/6 from GRT. By means of the quantization of adiabatic invariants in accordance with the method closed to the Bohr and Sommerfeld one without the Dirac equation the addition to the energy for the fine level splitting is obtained. It is shown that the Caplan's stable orbits in the hydrogen atom coincide with the Born orbits.
\end{abstract}

\section{KEYWORDS}

Field of the bound charges, general relativity theory (GRT), special relativity theory (SRT), equations of motion, quasi-classics, quantization of adiabatic invariants, Bohr, Sommerfeld, Caplan, Dirac, potential curve, level splitting, fine structure.

\section{INTRODUCTION}

In classic electrodynamics commonly thought that the field of rest point charge in the inertial reference frame (IRF) is the Coulomb spherically symmetric field independently of whether the charge is free or the sum of the forces affecting on the charge is equal to zero. However the field of this charge with uniformly accelerated motion in accordance with the classic electrodynamics for the noninertial reference frame (NRF) observer will be axially-symmetrical independently of the transition method to NRF. Thus, identical physical situation in which the charges are (identical constraint forces) results in the firelds with different symmetry!

Let us consider the charged metallic sphere. Each charge element is subjected to a force of negative pressure from the side of the field created with the charged sphere. This force is directed outward normally to the sphere surface. It is compensated with the crystalline force. The crystalline bonding force is directed inside the sphere and in accordance with the [1-7] the metrics 
or the charge element on the sphere will be axially-symmetrical with the "acceleration" directed inward the sphere

$$
d S^{2}=\exp \left(\frac{2 a_{0} y^{1}}{c^{2}}\right)\left(d y^{0}\right)^{2}-\left(d y^{1}\right)^{2}-\left(d y^{2}\right)^{2}-\left(d y^{3}\right)^{2},(1)
$$

where the "acceleration" $a_{0}$ is considered as positive if it is directed along the $y^{1}$ axis and this "acceleration" is negative if it is directed opposite this axis. We point out that for each charge element the local triad is selected so that $y^{1}$ axis coincides with the unit vector directed along the sphere radius. Let the sphere is charged negatively. Then the scalar potential from the charge element on the sphere has the form $[3,6,7]$

$$
d A_{0}=\frac{d Q}{r^{\prime}} \exp \left\{-\frac{a_{0} r^{\prime}(1-\cos \theta)}{2 c^{2}}\right\}
$$

where $r^{\prime}$ is the three-dimensional (Euclidean) distance from the charge $d Q$ up to the observation point, $\theta$ is the angle between the radius vector $\vec{r}^{\prime}$ and $\vec{i}, \vec{i}=\vec{a}_{0} /\left|\vec{a}_{o}\right| . \vec{i}$ for each charge element $d Q$ is directed the the sphere centre. The "acceleration" $a_{0}$ for the charges of the negatively charged sphere (electrons) is calculated in accordance with the formula

$$
a_{0}=\frac{e E}{2 m},(3)
$$

Here $e$ is the charge value, $m$ is the electron mass, $E$ is the field strength at the sphere surface. For the positively charged sphere the "relativistic" effect will be considerably smaller as the mass of the positive ion significantly exceeding the electron mass will be the $m$ mass. Therefore the field of the positively charged sphere in practice coincides with the classical field and for the negatively charged sphere the "relativistic" corrections can be significant. Each electron on the sphere surface belongs to tangent plane space but to the Riemann space-time. Therefore the integration on the sphere occurs in the plane space and it is correct. Integrating we obtain

$$
A_{0}=\frac{Q \exp \left(-\zeta^{2}\right) \sqrt{\pi} i}{4 R \zeta}[\Phi(i \zeta(1-2 R / r))-\Phi(i \zeta)], \quad \zeta=\frac{r}{R} \sqrt{\frac{e Q}{8 m c^{2} R}} .(4)
$$

In (4) $r$ is the distance from the sphere centre to the observation point, $\Phi(i \zeta)$ is the probability integral of imaginary argument determined as following

$$
\Phi(i \zeta)=\frac{2 i}{\sqrt{\pi}} \int_{0}^{\zeta} \exp \left(t^{2}\right) d t .(5)
$$

On the other hand the aggregation of the electrons at the sphere surface does not belong to the congruence of the world lines of NRF basis and it is included to the aggregation of the world lines belonging to the spherically symmetrical Lagrangian co-moving NRF with the metric

$$
d S^{2}=\exp (v)\left(d y^{0}\right)^{2}-r^{2}\left(d \theta^{2}+\sin ^{2} \theta d \phi^{2}\right)-\exp (\lambda)(d r)^{2} .(6)
$$

The expression for the metric coefficients is obtained in [2-6] and it has the form 


$$
\begin{aligned}
& \exp (v / 2)=1+\frac{q A_{0}}{m_{0} c^{2}} .(7) \\
& \exp \left(\frac{\lambda}{2}\right)=-\frac{\frac{r^{2}}{Q} \frac{\partial A_{0}}{\partial r}}{1+\frac{q A_{0}}{m_{0} c^{2}}} .(8)
\end{aligned}
$$

Thus, taking into account that the charges on the sphere are connected with the crystalline forces we obtained the spherically symmetric field of the charged sphere (but not the Coulomb one) specified in the Riemann space-time with the metric $(4,6-8)$.

\section{The Particle Motion in the Centrally Symmetric Electrostatic Field OF THE Bound CHARGES}

Let us consider the motion of the charged particle with $m_{0}$ mass and $q$ charge in the field which other $Q$ charge creates. The charge mass creating the field is great as compared with $m_{0}$ therefore we will consider $Q$ charge is immobile. In accordance with the idea of the general relativity theory the test charge will move on the geodetic line in the field of the bound charges forming the $Q$ charge with the metric (6-8).

To describe the particle motion instead of the geodetic equation it is convenient to use the Hamilton - Jacobi equation. It is known that when moving in the centrally symmetric field the particle path is in plane of the immobile charge centre creating the field. $\theta=\pi / 2$ plane is selected.

Hamilton - Jacobi equation will have the same form as in general relativity theory when describing the motion in the centrally symmetric gravitational field [8]

or we have

$$
g^{\mu \nu} \frac{\partial S}{\partial y^{\mu}} \frac{\partial S}{\partial y^{v}}-m_{0} c^{2}=0 .(9)
$$

$$
e^{-v}\left(\frac{\partial S}{\partial y^{0}}\right)^{2}-e^{-\lambda}\left(\frac{\partial S}{\partial r}\right)^{2}-\frac{1}{r^{2}}\left(\frac{\partial S}{\partial \phi}\right)^{2}-m_{0}^{2} c^{2}=0 .(10)
$$

In accordance with the general method of solution of Hamilton - Jacobi equation we represent $S$ in the form

$$
S=-\frac{E_{0} y^{0}}{c}+M \phi+S_{r}(r) .(11)
$$

Here $E_{0}$ is the constant energy, $M$ is the permanent angular momentum. From (10) and (11) we have

$$
S_{r}=\int \sqrt{\exp (\lambda-v) \frac{E_{0}^{2}}{c^{2}}-\left(m_{0}^{2} c^{2}+\frac{M^{2}}{r^{2}}\right) \exp (\lambda)} d r .(12)
$$


For integrand we have

$$
\begin{array}{r}
\exp (\lambda)=\frac{\frac{r^{4}}{Q^{2}}\left(\frac{\partial A_{0}}{\partial r}\right)^{2}}{\left(1+\frac{q A_{0}}{m_{0} c^{2}}\right)^{2}} .(13) \\
\exp (\lambda-v)=\frac{\frac{r^{4}}{Q^{2}}\left(\frac{\partial A_{0}}{\partial r}\right)^{2}}{\left(1+\frac{q A_{0}}{m_{0} c^{2}}\right)^{4}} .(14)
\end{array}
$$

Let us find the dependence of $r$ radial coordinate on $y^{0} / c$ universal time from equation

$$
\frac{\partial S}{\partial E_{0}}=\text { const. }(15)
$$

Using (11-14), we find

$$
\frac{v^{(1)}}{c}=\frac{\exp (\lambda / 2) d r}{\exp (v / 2) d y^{0}}=\sqrt{1-\frac{m_{0}^{2} c^{4}}{E_{0}^{2}}\left(1+\frac{M^{2}}{m_{0}^{2} c^{2} r^{2}}\right)\left(1+\frac{q A_{0}}{m_{0} c^{2}}\right)^{2}} .(16)
$$

Here $v^{(1)}$ is the radial "physical" component of three-dimensional velocity.

For example let us consider the fall of the negatively charged particle on the positively charged coulomb centre.

From (16) at $M=0$ we find

$$
\frac{v^{(1)}}{c}=\sqrt{1-\frac{m_{0}^{2} c^{4}}{E_{0}^{2}}\left(1+\frac{q A_{0}}{m_{0} c^{2}}\right)^{2}} \cdot(17)
$$

As previously shown for positive charges the "relativistic" corrections stipulated for the "acceleration" of bound charges creating the field give a small correction to the scalar potential. Therefore

$$
\frac{v^{(1)}}{c}=\sqrt{1-\frac{m_{0}^{2} c^{4}}{E_{0}^{2}}\left(1-\frac{|q \| Q|}{m_{0} c^{2} r}\right)^{2}}
$$

Let us consider the problem concerning the radial electron motion in the proton field without quantum effects. As is known from the nuclear physics the charge distribution radius inside the proton is $R=0.8 \cdot 10^{-13} \mathrm{~cm}$. The classic electron radius is $r_{0}=2.8 \cdot 10^{-13} \mathrm{~cm}$. The scalar potential outside the proton can be calculated in accordance with the formula (4). 


$$
A_{0}=\frac{Q \exp \left(-\zeta^{2}\right) \sqrt{\pi} i}{4 R \zeta}[\Phi(i \zeta(1-2 R / r))-\Phi(i \zeta)], \zeta=\frac{r}{R} \sqrt{\frac{e Q}{8 m c^{2} R}}, \delta=\sqrt{\frac{e Q}{8 m c^{2} R}}(19) .
$$

Outside the proton the relation is valid

$$
\zeta=\frac{r}{R} \sqrt{\frac{e Q}{8 m c^{2} R}}=\frac{r}{R} \sqrt{\frac{m_{e} r_{0}}{8 m_{p} R}}=0.0154 \frac{r}{R} .(20)
$$

In the last formula $m_{e}, m_{p}$ are the electron and proton masses respectively. Taking into account the infinitesimality $\delta=0.0154$ and expanding (19) into the Taylor series we obtain for the formula (17)

$$
\frac{v^{(1)}}{c}=\sqrt{1-\frac{m_{0}^{2} c^{4}}{E_{0}^{2}}\left(1-\frac{r_{0}}{r}\right)^{2}} \cdot(21)
$$

Let the electron had zero speed at infinity that is equivalent to the relation $E_{0} / m_{0} c^{2}=1$. In this case from formula (21) we have

$$
\frac{v^{(1)}}{c}=\sqrt{1-\left(1-\frac{r_{0}}{r}\right)^{2}} \cdot(22)
$$

As follows from (22) when fall on the proton firstly the electron velocity increases from zero up to light speed $c$ reaching the later at the diatance from the proton centre equal to the classic electron radius $r_{0}$. Further when approaching to the centre the electron velocity begins to decrease ant it vanishes at the distance from the proton centre equal to the half of the classic electron radius $r_{0} / 2$. Then when decreasing of the radius the radicand becomes negative and the velocity becomes imaginary that is devoid of physical meaning. Thus, the obtained solution is valid when $r \geq r_{0} / 2$.

The decreasing of the "physical" electron velocity is equivalent to the repulsion from the proton side at the small distances $1.4 \cdot 10^{-13} \mathrm{~cm}$ which are approximately equal to the effective proton radius.

To compare we point out that in the similar problem when moving on the particle radius in the gravitational field specified with the Schwarzshil'd metric the "physical" velocity is determined with the formula [9]

$$
\frac{v^{(1)}}{c}=\sqrt{1-\left(1-\frac{r_{g}}{r}\right)}=\sqrt{\frac{r_{g}}{r}}
$$

In (23) $r_{g}$ is the gravitational radius.

When formal substitution $e^{2} \rightarrow k m_{p} m_{e}$ or $r_{0} \rightarrow r_{g} / 2$ the solutions (22) and (23) in the distance from the gravitational radius coincide. Near the gravitational radius these solutions are qualitatively differed from one another. In (23) the "physical" particle velocity increases with the 
radius decrease, achieves the light velocity $c$ at the gravitational radius and it goes to infinity at $r \rightarrow 0$.

In our case (22) the value of the particle velocity like in SRT does not exceed the velocity of light in free space achieveing the later at $r=r_{0}$.

The solution of the similar problem in SRT [8] results in the relation

$$
\frac{v^{(1)}}{c}=\sqrt{1-\frac{1}{\left(1+\frac{r_{0}}{r}\right)^{2}}} \cdot(24)
$$

As follows from (24) the electron velocity when approaching to the centre increases with the radius decrease and it tends to the velocity of light $c$ at $r \rightarrow 0$. At $r=r_{0}, v^{(1)}=0.866 c$. It is clear that in our consideration at $r>r_{0}$ the maximum deviation of the velocity from the SRT solution does not exceed 14\%. At $r_{0} / r=1$ the solutions (22) and (24) coincide. At $r_{0} / r>1$ the velocity behaviour is changed: in our case it decreases and in SRT it increases.

Let us investigate the radial motion of ultrarelativistic electrons which even at infinity have infinitely large energy. Supposing in (17) $E_{0} \rightarrow \infty$ we obtain for any $r, v^{(1)}=c$. For similar case the same result will be obtained in SRT and GRT.

Let us investigate more detailed the relation (21) introducing the dimensionless electron energy $E$ and dimensionless velocity $v$ in accordance with the formula

$$
E=\frac{E_{0}}{m_{e} c^{2}}, \quad v=\frac{v^{(1)}}{c}
$$

According to agreed notations we have

$$
v=\sqrt{1-\frac{1}{E^{2}}\left(1-\frac{r_{0}}{r}\right)^{2}} \cdot(26)
$$

As follows from (21) for any $E \geq 1$ value $v$ has a maximum equal to unity at the point $r=r_{0}$. For greater energy values the velocity growth curve from infinity to $r_{0}$ point will be more smooth than for smaller energy values. For ultrarelativistic particles the growth curve coincides with the straight line $v=1$. After maximum the velocity curve will fall and at the some radius value $v$ goes to zero. The distance $r_{\min }$ corresponding to zero valuation of the velocity will be the closely to the Coulomb centre, the more the particle energy is. It follows from the formula

$$
r_{\min }=\frac{r_{0}}{E+1} .(27)
$$

It follows from (27) that only electrons with infinitely large energy $E$ reach the Coulomb centre. However it is not the case. Formulas (26), (27) are used for the proton with the finite radius. For 
the model point proton one should use the general fomulas (17) and (4). In accordance with (4) for the scalar potential near the coulomb centre the formula holds

$$
A_{0}(R)=\frac{Q \exp \left(-\delta^{2}\right) \sqrt{\pi}}{2 R i \delta}[\Phi(i \delta)],(28)
$$

whence we find at $z=\delta>>1$ the expression

$$
A_{0}(R)=\frac{4 m c^{2}}{e}
$$

It follows from the last formula and (17) that the electrons with energy (30) can have zero velocity at the Coulomb centre

$$
E=4 \frac{m_{p}}{m_{e}}-1 .(30)
$$

Thus, electrons with high but final energy can reach the point Coulomb centre.

Let us elucidate the physical meaning of the origin of the electron stop in the proton field. Equating in (26) the radicand to zero we find the function $E(r)$ acting as the potential curve in the nonrelativistic theory.

$$
E(r)=\left|1-\frac{r_{0}}{r}\right| .(31)
$$

It follows from the analysis (31) that as a result of the presence of the modulus the $E(r)$ function at $r<r_{0}$ does not damp but it increases and becomes equal to unit at $r=r_{0} / 2$, and this function goes to infinity at $r \rightarrow 0$. Thus, when the distances are smaller then $r=r_{0}$ the "effective repulsion" takes place. This repulsion results in the electron stop at $r=r_{\min }$ in accordance with (27). As $E(\infty)=1$ the potential well with single depth is formed. At $E=E_{1}<1$ the radial motion of the electron in the proton field is finite. The electron radially oscillates within the limits from $r_{\min }$ up to $r_{1}=r_{0} /\left(1-E_{1}\right)$. At $E=E_{1}>1$ the motion is infinite. The electron reaching when fall the $r_{\min }$ value shifts at infinity after the "reflection".

In SRT the solution of such problem at $M=0$ results in the fall of the charge on the coulomb centre. Similar situation takes place with zero moment in the Newtonian mechanics and in GRT

Let us find the electron motion on the circular orbits in the proton field. Assuming in (16) $v^{(1)}=0$ that corresponds to the circular orbits at $M \neq 0$ we obtain the expression for the potential curve $U(r)=E_{0}(r) /\left(m_{e} c^{2}\right)$

$$
U=\sqrt{1+\frac{M^{2}}{m_{e}^{2} c^{2} r^{2}}}\left|1-\frac{r_{0}}{r}\right| .(32)
$$

The considered problem is similar to known S. A. Caplan problem in GRT [8], [9]. To compare with the Caplan solution we make a formal substitution $r_{0} \rightarrow r_{g} / 2$. The meaning of this substitution is as follows. After the substitution the approximate metric $(7,8)$ after the Taylor 
expansion coincides with the exact GRT Schwarzshil'd metric. The circular orbits in the Caplan problem are determined in the Schwarzshil'd field.

For simplicity we introduce the dimensionless $E$ energy values (25), $a$ moment and $x$ dimensionless radius in accordance with the formulas

$$
a=\frac{M}{m_{e} c r_{g}}, \quad x=\frac{r}{r_{g}}, \quad r_{0} \rightarrow \frac{r_{g}}{2} .(33)
$$

After substitution the relation (32) has the form

$$
U=\sqrt{1+\frac{a^{2}}{x^{2}}}\left|1-\frac{1}{2 x}\right| .(34)
$$

The radiuses of the circular orbits and corresponding energy and moment values are determined by the extremums of the effective potential energy $U(x)$. Minimum of the function corresponds to the stable circular orbits and maximum corresponds to the unstable orbits. Solving jointly the equations set $U(x)=E$ and $U^{\prime}(x)=0$ we obtain

$$
x_{1,2}=a^{2}\left(1 \pm \sqrt{1-\frac{2}{a^{2}}}\right), \quad E=\frac{\sqrt{2} a}{\sqrt{x_{1,2}}}\left|1-\frac{1}{2 x_{1,2}}\right|^{3 / 2} .
$$

Plus sign in the formula for the orbit radiuses corresponds to the stable orbit and minus sign corresponds to the unstable one. In order to compare we present the solution of the Caplan problem in our designations

$$
x_{1,2}=a^{2}\left(1 \pm \sqrt{1-\frac{3}{a^{2}}}\right), \quad E=\frac{\sqrt{2} a}{\sqrt{x_{1,2}}}\left|1-\frac{1}{x_{1,2}}\right|(36)
$$

and the expression of the effective potential $U_{0}(x)$ in GRT.

$$
U_{0}(x)=\sqrt{\left(1+\frac{a^{2}}{x^{2}}\right)\left(1-\frac{1}{x}\right)} .
$$

Iit is clear if we introduce in (34) radicand the expression with the modulus and expand into Taylor series limiting with the first expansion term we will obtain the potential curve (37). However this is correct only for $x>>1$. It follows from the (37) analysis this expression is determined only for the values $x \geq 1$. If not $U_{0}$ becomes an imaginary one.

For (34) such imitations do not exist. Potential curve charts (34) for different values of $a$ moment are presented in [8], [9] and for our case potential curve charts are presented in [3]. Curve charts (34) at different $a$ in $x \geq 1$ are similar with the charts (37). They are differed only with maximum and minimum. You could see that with the comparison the expressions (35) and (36). 
For example, at $a^{2}=2$ maximum and minimum coordinates for our case coincide. In GRT it takes place at $a^{2}=3$. For our case the nearest to the centre stable circular orbit corresponds to the value $r_{1}=2 r_{g} \rightarrow 4 r_{0}$. Corresponding energy is $E_{1}=\sqrt{27 / 32}=0.919$, and the velocity of the circular motion is determined from the formula

$$
E_{0}=\frac{m_{0} c^{2} \sqrt{g_{00}}}{\sqrt{1-\frac{v^{2}}{c^{2}}}} .(38)
$$

of the energy conservation law which is similar to the energy conservation law for the particle in the constant gravitational field in GRT [8]. In (38) the velocity $v$ is calculated in accordance with the formula

$$
v=\frac{c d l}{\sqrt{g_{00}} d y^{0}},(39)
$$

$v=c \sqrt{3} / 3=0.577 c$.

In GRT we have respectively $r_{1}=3 r_{g}, E_{1}=\sqrt{8 / 9}=0.943, v=c / 2$ [9].

At $a^{2}<2$ for our case the curve does not have the extremums (in GRT for $a^{2}<3$ ). With the growth of the $a$ moment from $\sqrt{2}$ to $\infty$ the coordinates of maximums are decreased from $r_{2}=4 r_{0}$ to $r_{2}=2 r_{0}$ (in GRT from $r_{2}=3 r_{g}$ to $r_{2}=1.5 r_{g}$ ). $E_{\max }$ energy is increased from $E_{\max }=0.919$ to $E_{\max }=\infty$ (in GRT from $E_{\max }=0.943$ to $E_{\max }=\infty$ ).

The nearest to the centre unstable circular orbit corresponds to the value $r_{1}=r_{g} \rightarrow 2 r_{0}$, $E_{\max }=\infty, v=c\left(\right.$ in GRT $\left.r_{1}=1.5 r_{g}, E_{\max }=\infty, v=c\right)$.

In fig. 1 four dimensionless potential curves $U(x, a)$ for different values of the dimensionless $a$ moments in the Caplan problem in the Coulomb field of the bound charges are presented. The lowest curve corresponds to $a=0$ and it does not have extremums.

The second curve from the bottom corresponds $a=\sqrt{2}$ when the coordinates of maximum and minimum of the potential curve coincide. The third curve from the bottom has maximum equal to unit at $a=1.665$, and the fourth curve at $a=5$ has maximum equal to 2.551 . 


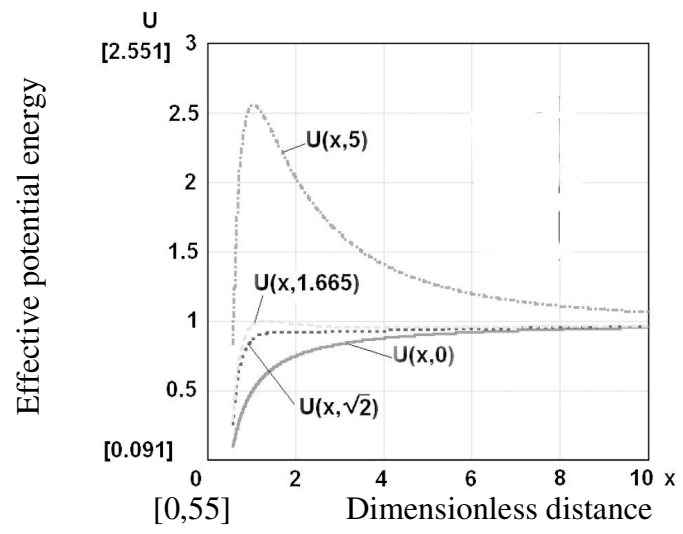

Figure 1. Dimensionless potential curves $U(x, a)$ for different values of the dimensionless $a$ moments in the Caplan problem in the Coulomb field of the bound charges

In fig. 2 the curve showing the arms of the stable and unstable orbits in the Caplan problem for the field of the bound charges is presented. The lower reach determines the radiuses of the unstable orbits, and upper reach determines the radii of the stable orbits. The curve calculation is carried out in accordance with the formula (35).

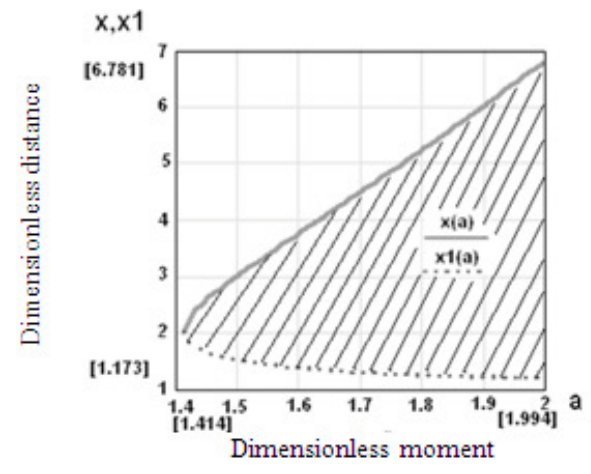

Figure 2. Curve of the dependence of dimensionless distance $x$ on $a$ moment in the Caplan problem in the Coulomb field of the bound charges

It should be noted that in the Newton mechanics at the centrally symmetric gravitational field the stable circular orbits exist at any distance from the centre. In the Coulomb field of the bound charges the minimum radius $a=\sqrt{2}$ exists. At smaller radius circular orbits do not exist. Similar restriction takes place in GRT where minimum radius is $a=\sqrt{3}$.

Although outwardly the Caplan solution and our one do not greatly differ, however the important basic difference takes place. The presence of the expression with the modulus in the effective potential energy $U(x)$ in (34) results in the additional minimum value $U(x)$ in the $x=1 / 2$ point. In is clear that $x$ derivative from the $U(x)$ function in this point has a discontinuity as at the radial fall of the particle that is at $a=0$. In $x=1 / 2$ point the attraction changes on the repulsion and $U(x)$ function unrestrictedly increases when the radius vanishes. The presence of the additional centrifugal energy makes the function increase $U(x)$ faster when vanishing than at 
the zero moment. Aforesaid is shown in fig. 3 where the plot of function (34) is continued to $\tilde{\mathrm{o}}<0.5$.

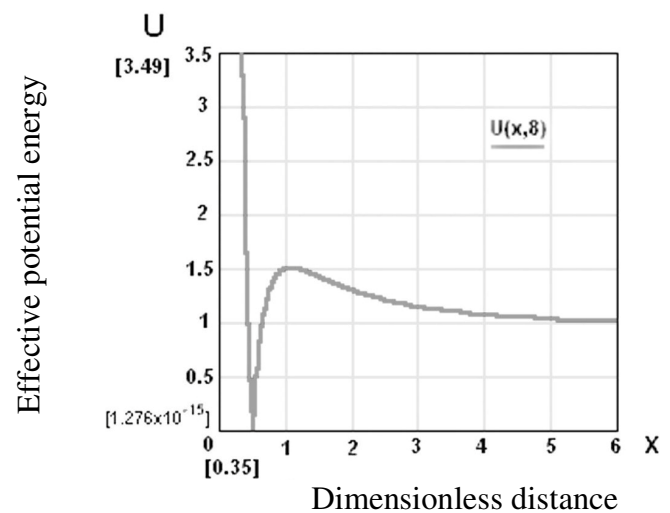

Figure 3. Potential curve in the Coulomb field of the bound charges

The presence of the maximum at the potential curve in the Schwarzshil'd field results in the gravitational capture if the particle energy is $E>E_{\max }$. For our case at $E>E_{\max }=1.5$ the capture does not occur as any $E$ value always "meet" the potential curve at $0.5>x>0$ (see fig. $3)$. We remind that the dimensionless value $x$ on the $\mathrm{X}$-axis is the ratio $x=r / r_{g} \rightarrow r /\left(2 r_{0}\right)$. Thus, similarly to the Newton mechanics at any high energy the particle rounds the attractive centre and goes into infinity. Even as opposed to the Newton mechanics it takes place at the radial fall on the Coulomb centre too. It is significant to note that the stable electron equilibrium in the proton field at $x=1 / 2 \rightarrow r=r_{0}$ exists. In the classic consideration it is absent.

The presence of the additional "potential well" with minimum value $U\left(r_{0}\right)=0$ (see fig. 3) permits the existence of the finite electron motion in this well. Thus, the proton and the electron can form the stable connection "neutron" with the dimensions of the order of $r_{0}$. Of course the considered approach has only a methodological interest as the quantum effects must already become apparent at the distances considerably greater $r_{0}$.

Let us consider the electron trajectory at the proton field. As is known [8] the trajectory is determined with the equation $\partial S / \partial M=$ const . Whence we have

$$
\phi=\int \frac{M d r}{r^{2} \sqrt{\exp (\lambda-v) \frac{E_{0}^{2}}{c^{2}}-\left(m_{0}^{2} c^{2}+\frac{M^{2}}{r^{2}}\right) \exp (\lambda)}} \text {. (40) }
$$

It is of the methodological interest to investigate the electron trajectories in the atom limits similarly to the planetary motion in the sun gravitational field for the electron motion in the proton field at the distances compared with the atom dimensions $r \gg>r_{0}$. Therefore the proposed theory must only result in insignificant correction data as compared with the general Coulomb interaction.

To calculate the correction data to the trajectory we will assume as in [8] from the radial part of the action before the moment of its $M$ differentiation (12-14). 


$$
S_{r}=m_{0} c \int \sqrt{\frac{E^{2} r^{4}}{\left(r-r_{0}\right)^{4}}-\frac{M^{2}}{m_{0}^{2} c^{2}\left(r-r_{0}\right)^{2}}-\frac{r^{2}}{\left(r-r_{0}\right)^{2}}} d r .(41)
$$

After the transformation of integration variable

using easily verifiable formula

$$
r-r_{0}=r^{\prime}
$$

$$
m_{0}^{2} c^{2}\left(E^{2}-1\right)=2 m_{0} E^{\prime}+\frac{E^{\prime 2}}{c^{2}}
$$

where $E^{\prime}$ is the nonrelativistic particle energy (without the rest energy) we obtain with the required precision the expression

$$
S_{r}=\int \sqrt{2 m_{0} E^{\prime}+\frac{E^{\prime 2}}{c^{2}}+\frac{2 r_{0} m_{0}^{2} c^{2}+8 r_{0} m_{0} E^{\prime}}{r}-\frac{M^{2}-5 r_{0}^{2} m_{0}^{2} c^{2}}{r^{2}}} d r .
$$

When formal substitution $e^{2} \rightarrow k m_{p} m_{e}$ or $r_{0} \rightarrow r_{g} / 2$ the structure of the radial part of the action $S_{r}$ is close to the similar value used to describe the planetary motion in the centrally symmetric gravitational field in GRT. The difference is revealed only in the last factor at $1 / r^{2}$. In GRT $6 r_{0}^{2} m_{0}^{2} c^{2}$ value is subtracted from $M^{2}$ (in our designations). The rest of the terms coincide.

As is known [8] the correction factors in the first two terms of the radicand are only influenced by the changing of the connection between the energy, particle moment and parameters of its orbit. The factor subtracted from $M^{2}$ results in the systematic drift of the orbit perihelion. Considering that $5 r_{0}^{2} m_{0}^{2} c^{2} / r^{2}$ term is the small small correction relatively $M^{2} / r^{2}$ after the expansion of the intergrand into a series we obtain the relation

$$
S_{r}=S_{r}^{0}+\delta S_{r}
$$

where $S_{r}^{0}$ corresponds (42) at zero correction, and $\delta S_{r}$ is determined with the equality

$$
\delta S_{r}=\int \frac{5 m_{0}^{2} c^{2} r_{0}^{2} d r}{2 r^{2} \sqrt{2 m_{0} E^{\prime}+\frac{E^{\prime 2}}{c^{2}}+\frac{2 r_{0} m_{0}^{2} c^{2}+8 r_{0} m_{0} E^{\prime}}{r}-\frac{M^{2}}{r^{2}}}}
$$

Increment $\Delta \delta S_{r}$ during the orbital period of the electron in accordance with the nonrelativistic mechanics at the Coulomb field after the integration in (44) will have the form

$$
\Delta \delta S_{r}=\frac{5 \pi m_{0}^{2} c^{2} r_{0}^{2}}{M}
$$

As the trajectory is determined with the equation 
then during the period we have

$$
\frac{\partial S}{\partial M}=\phi+\frac{\partial S_{r}}{\partial M}=\text { const }
$$

$$
\Delta \phi=-\frac{\partial \Delta S_{r}}{\partial M}=-\frac{\partial \Delta S_{r}^{0}}{\partial M}-\frac{\partial \Delta \delta S_{r}^{0}}{\partial M} .
$$

Using (45) and taking into account that

$$
-\frac{\partial \Delta S_{r}^{0}}{\partial M}=2 \pi
$$

we obtain

$$
\Delta \phi=2 \pi+\frac{5 \pi m_{0}^{2} c^{2} r_{0}^{2}}{M^{2}}
$$

Second term in (46) determines the perihelion shift of the electron orbit around the proton. The solution of this problem in SRT results in the perihelion shift five times smaller than our shift and six times smaller than in GRT at the formal substitution $e^{2} \rightarrow k m_{p} m_{e}$ or $r_{0} \rightarrow r_{g} / 2$. Thus, the developed apparatus is much closer to GRT than SRT.

Let us consider the nonradial motion of the ultrarelativistic electrons in the proton field (analog of the light ray propagation in the centrally symmetric gravitational field). We consider by definition that even at infinity $v_{\infty} \rightarrow c$.

The radial part of the action (41) after the transformation of integration variable

$$
r-r_{0}=r^{\prime}
$$

provided $E \gg>1, E_{0}=m_{0} c^{2} E$ and the impact parameter $\rho$ is connected with the $M$ moment in accordance with the formula [1]

$$
\rho=\frac{M}{m_{0} c E}(47)
$$

is reduced to the form

$$
S_{r}=\frac{E_{0}}{c} \int \sqrt{1+\frac{4 r_{0}}{r^{\prime}}-\frac{\rho^{2}}{r^{\prime 2}}+\frac{6 r_{0}^{2}}{r^{\prime 2}}} d r .(48)
$$

Firstly let us conside the case when $r_{0} / \rho=1$. For this case (48) has the form

$$
S_{r}=\frac{E_{0}}{c} \int \sqrt{1+\frac{4 r_{0}}{r^{\prime}}-\frac{\rho^{2}}{r^{\prime 2}}} d r .
$$

The last expression accurate within the multiplier and substitution $2 r_{0} \rightarrow r_{g}$ coincides with the radial part of the eikonal for the light ray propagation at the gravitational field in GRT [8]. Using the derivation [8] we obtain that under the influence of the Coulomb attraction field from the 
proton the trajectory of the ultrarelativistic electron is curved forming the curve with the concavity towards the centre. The angle between two asymptotes of this curve is differed from $\pi$ by $\delta \phi$ determined from the equality

$$
\delta \phi=\frac{4 r_{0}}{\rho} .(50)
$$

Let us analyzed the motion of the ultrarelativistic electrons when the impact parameter $\rho$ is of the same order of $r_{0}$. To analyze the motion we use the formula (16) which at $E \gg 1$ is reduced to the form

$$
\frac{v^{(1)}}{c}=\sqrt{1-\frac{\rho^{2}}{r^{2}}\left(1-\frac{r_{0}}{r}\right)^{2}} \cdot(51)
$$

Obviously the approaching of the electron to the proton will stop when the radial velocity component $v^{(1)}=0$. The turning point will be determined from zero radicand in (51).

This results in the relation between the impact parameter and the turning point coordinate represented in the form

$$
\rho_{1}=\frac{r_{1}^{2}}{\left|r_{1}-1\right|}, \quad \rho_{1} \equiv \frac{\rho}{r_{0}}, \quad r_{1} \equiv \frac{r}{r_{0}} .(52)
$$

The curve (52) has minimum in point

$$
r_{1}=2, \quad \rho_{1 \min }=4(53)
$$

and two asymptotes: the vertical one $r_{1}=1$ and the slanting line $Y=r_{1}+1$. The presence of the modulus in the denominator expands the field of the variable existence up to the values $r_{1} \rightarrow 0$.

The second branch of a curve appears which when variating of the argument from $r_{1}=1$ to $r_{1}=0$ changes respectively from $\infty$ to zero. Using near the Coulomb centre the formula (28) for zero component of 4 - potential in (16) influences only on zero approximation behavior at $r_{1} \rightarrow 0$. The dependence of $R 1 \equiv \rho_{1}$ on $r_{1} \equiv r 1$ is presented in fig. 4 . 


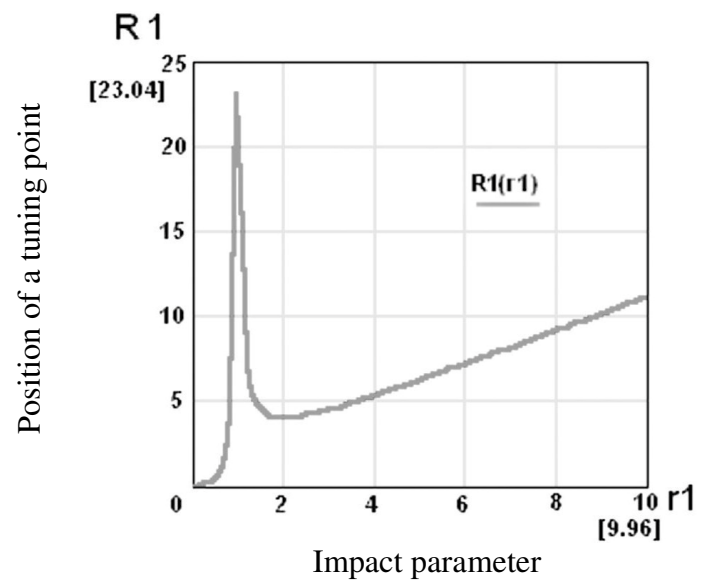

Figure 4. Curve of dependence of $r_{1}$ ultrarelativistic particle on $\rho_{1}$ impact parameter in the Coulomb field of the bound charges.

The particles with $\rho_{1}<4$ suffer the "pseudotrapping"

To analyze the solution obtained we write the similar equation for the curve in GRT for the ultrarelativistic particle in the Schwarzshil'd field. In accordance with [8] and [9] for the desired curve we have

$$
\rho_{2}=\frac{r_{2}^{3 / 2}}{\sqrt{r_{2}-1}}, \quad \rho_{2} \equiv \frac{\rho}{r_{g}}, \quad r_{2} \equiv \frac{r}{r_{g}} .(54)
$$

The curve (53) has minimum in point

$$
r_{2}=\frac{3}{2}, \quad \rho_{2 \min }=\frac{3 \sqrt{3}}{2}(55)
$$

and two asymptotes: the vertical one $r_{2}=1$ and the slanting line $Y=r_{2}$.

Both considered curves at $r_{1}>1$ and $r_{2}>1$ are similar. They are differed from only with minimums and their minimal values. However at $r_{2}<1$ the curve (53) is not determined as the radicand becomes negative. Thus, as opposed to our case in GRT the second branch of a curve is absent. In GRT the ultrarelativistic particle flying from infinity with the impact parameter $\rho_{2}<\rho_{2 \min }=2.6$ does not meet the turn curve and consequently its gravitational capture occurs. The capture cross-section is determined with the formula

$$
\sigma=\pi \rho_{2 \min }^{2} r_{g}^{2}=\frac{27 \pi r_{g}^{2}}{4}=6.75 \pi r_{g}^{2} .(56)
$$

In our case at $\rho_{1}<\rho_{1 \min }=4$ the electron "passes" under the first turn curve but "meets" the second branch of this curve (see fig. 4). The peak value of the point of contact coordinate with the second branch is determined from the apparent equation 


$$
\rho_{1 \text { min }}=\frac{r_{1}^{2}}{1-r_{1}},(57)
$$

The solution of this equation has the form

$$
r_{1 \max }=\frac{\sqrt{\rho_{1 \min }^{2}+4 \rho_{1 \min }}-\rho_{1 \min }}{2}=0.83 .(58)
$$

As opposed to GRT the electron "flying" under the turn curve is not captured with the proton because of the proton repulsion prevents. This repulsion appears at the distances $r<r_{0}$.

From $r=2 r_{0}$ up to $r=r_{0}$ distances the electron trends to the capture. Therefore we denote the pseudotrapping cross- section by $\sigma_{p}$

$$
\sigma_{p}=\pi \rho_{1 \min }^{2} r_{0}^{2}=16 \pi r_{0}^{2} \rightarrow 4 \pi r_{g}^{2} .(59)
$$

Let us consider one more case when the electron at infinity has a negligible velocity $v_{\infty}$ as compared with the velocity of light $c$ that corresponds to $E=1$.

In GRT the section of the gravitational capture is determined from the requirement of the maximal value of the potential curve (37) $U\left(x_{\max }\right)=1 . x_{\max }=2, a=2$ correspond to this energy value. The impact parameter $\rho=2 c r_{g} / v_{\infty}$ and the capture cross-section

$$
\sigma=\pi \rho_{2 \min }^{2} r_{g}^{2}=4 \pi r_{g}^{2}\left(\frac{c}{v_{\infty}}\right)^{2} .(60)
$$

All particles with $\rho<2 c r_{g} v_{\infty}$ are captured gravitationally.

In our case the potential curve $U(x)$ is specified with the relation (34). Solving jointly the system of equations $U(x)=1$ and $U^{\prime}(x)=0$ we obtain for the maximum (see fig. 1 , the second curve from the top)

$$
x_{1}=a^{2}\left(1-\sqrt{1-\frac{2}{a^{2}}}\right), \quad 1=\frac{\sqrt{2} a}{\sqrt{x_{1}}}\left|1-\frac{1}{2 x_{1}}\right|^{3 / 2} .(61)
$$

From (61) we find

$$
a^{2}=\frac{22+\sqrt{22^{2}+16}}{16}=2.773, \quad a=1.665, \quad x_{1}=1.309 .(62)
$$

The impacr parameter in our designations $\rho=3.33 \mathrm{cr}_{0} / v_{\infty}$ and the pseudotrapping cross- section

$$
\sigma_{p}=\pi \rho^{2}=11.1 \pi r_{0}^{2}\left(\frac{c}{v_{\infty}}\right)^{2} \rightarrow 2.77 \pi r_{g}^{2}\left(\frac{c}{v_{\infty}}\right)^{2} .(63)
$$


In our case the proton can not capture the electron so far as at $r<r_{0}$ the potential curve begins to grow and the electron energy $E=1$ will "intersect" with this curve (see fig. 5).

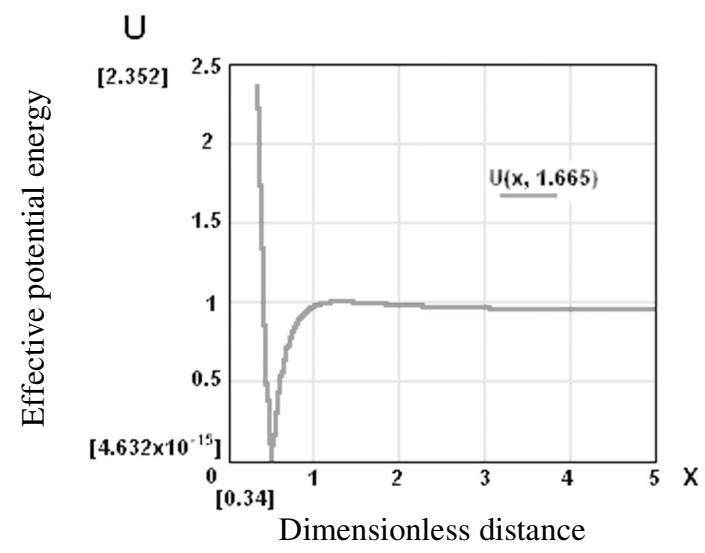

Figure5. Potential curve for the nonrelativistic particle having at infinity negligible velocity.

As opposed to GRT such particle will not be captured with the proton as it "meets" with an increasing branch of the curve after the passing of the maximum

It is known from the classical relativistic electrodynamics when moving in the Coulomb field [8] that at $M c<|Q e|$ and $Q e<0$ the particle trajectories represent the spirals with the fanishing radius $r$ at the angle $\phi \rightarrow \infty$. The fall time of the particle at origin is the finite quantity. Particularly for the nonrelativistic particle at infinity (the electron in the proton field) the condition of the fall on the centre is equivalent to the equality

$$
\rho<\frac{c r_{0}}{v_{\infty}}(64)
$$

In our case the impact parameter when pseudotrapping is 3.33 times as much than in SRT. A significant feature of our solution as opposed to SRT and GRT is the possibility of the stable static balance of the electron in the proton field at the distance $r_{0}$ from the proton centre. The radial oscillations relatively $r_{0}$ are allowed. This permits the (qualitative) possibiliy of the existence of the neutral stable particle with the dimensions of the order of $r_{0}$.

\section{Quantization of Adiabatic Invariants in Central Field of BOUND CHARGES}

It is of interest to consider the elementary possibilities of the accounting of quantum effects. As is known Bohr and Sommerfeld explained the spectrum of the hydrogen atom by means of the quantization of adiabatic invariants. Then Sommerfeld had attempted to take into account relativistic corrections in the network of the mechanical analog. He permitted that relativistic corrections can explaine the splitting of terms degenerated in the nonrelativistic theory. Thus, Sommerfeld wanted to create a theory of fine structure. 
It should be noted that in a way he pulled it off and he obtained formulas for the fine structure of hydrogen atomic levels in the old Bohr theory before the creation of the quantum mechanics without the solution of the Dirac equation.

The structure of the proposed approach is close to the Bohr and Sommerfeld one but it has the distinctions of kind.

1. As opposed to the Sommerfeld approach using the motion of the electron in the proton field in the SRT flat space-time we are working in the Riemann geometry stipulated for the field of elements of proton bound charges.

2. The world line of the electron in the proton field in the Sommerfeld theory corresponds in our case to the electron geodesic line in the Riemann space-time. In our approach the proton field is absent in explicit form. It becomes apparent in the form of the cuved space-time geometry.

As the atom dimensions are of the order of $10^{-8} \mathrm{~cm}$ and the nuclear sizes are of the order of $10^{-13} \mathrm{~cm}$ then factors of the metric (6) can be represented by means of $(7,8)$ in the form

$$
\exp (v)=\left(1-\frac{e^{2}}{r m_{0} c^{2}}\right)^{2}=\left(1-\frac{r_{0}}{r}\right)^{2}=\exp (-\lambda),(65)
$$

where $e$ is the electron charge, $m_{0}$ is the electron mass.

As is known [8], the 4-vector determined with the equality is called the generalized momentum $P_{\mu}$

$$
P_{\mu}=-\frac{\partial S}{\partial y^{\mu}} .(66)
$$

Let us calculate the radial component of the 4-pulse of the electron in the proton field. From (11) and (12) we find

$$
P_{r}=-\sqrt{\exp (\lambda-v) \frac{E_{0}^{2}}{c^{2}}-\left(m_{0}^{2} c^{2}+\frac{M^{2}}{r^{2}}\right) \exp (\lambda)} .(67)
$$

As opposed to SRT we apply the quantum condition to the "physical" radial component of 4pulse determined with tetrads. The expression for these tetrads has the form [2-4]. Tetrad indices are parenthesized.

$$
\begin{gathered}
e_{(\alpha)}^{\mu}=\frac{\delta_{\alpha}^{\mu}}{\sqrt{\left|g_{\alpha \alpha}\right|}}, \quad e_{\mu}^{(\alpha)}=\delta_{\mu}^{\alpha} \sqrt{\left|g_{\alpha \alpha}\right|}, \quad e_{\mu}^{(0)}=V_{\mu}, \quad e_{(0)}^{\mu}=V^{\mu},(68) \\
P_{(r)}=-\frac{1}{\sqrt{-g_{11}}} P_{r}=-\sqrt{\exp (-v) \frac{E_{0}^{2}}{c^{2}}-\left(m_{0}^{2} c^{2}+\frac{M^{2}}{r^{2}}\right)}=-P^{(r)} \text {.(69) }
\end{gathered}
$$

Following to Bohr and Sommerfeld we state quantization conditions in the form

$$
\int P^{(r)} d r=n_{r} h, \quad \oint M d \phi=n_{\phi} h,(70)
$$

where $n_{r}$ and $n_{\phi}$ are integral numbers. As a result we have the equality 


$$
\int \sqrt{\left(1+\frac{r_{0}}{r}\right)^{2} \frac{E_{0}^{2}}{c^{2}}-\left(m_{0}^{2} c^{2}+\frac{n_{\phi}^{2} \hbar^{2}}{r^{2}}\right)} d r=n_{r} h, \quad \hbar=\frac{h}{2 \pi} .(71)
$$

In this equality after the integration we identify $E_{0}$ values depending on quantum numbers $n_{r}$ and $n_{\phi}$. The integral can be represented in the form

$$
\begin{gathered}
\int \sqrt{-A+2 B / r-C / r^{2}} d r, \quad A=\left(m_{0}^{2} c^{2}-\frac{E_{0}^{2}}{c^{2}}\right), \\
B=\frac{r_{0} E_{0}^{2}}{c^{2}}, \quad C=n_{\phi}^{2} \hbar^{2}-\frac{r_{0}^{2} E_{0}^{2}}{c^{2}} .(72)
\end{gathered}
$$

As we consider the finite motion of the electron in the proton field the $A$ value is obviously negative. The integrand has two roots for positive $r$ values that obviously corresponds to the perihelion and the aphelion of the electron orbit. The integration is expected to be carried out from one root up to the other and vice versa with the changing of the sign before the root in the integrand. The similar problem with other constants has been solved in [11]. On the basis of which we have

$$
\int \sqrt{-A+2 B / r-C / r^{2}} d r=2 \pi\left(\frac{B}{\sqrt{A}}-\sqrt{C}\right) .(73)
$$

As a result we obtain the following equation for the determination of energy levels

$$
\frac{r_{0} E_{0}}{c^{2}} \frac{1}{\sqrt{m_{0}^{2} c^{2}-E_{0}^{2} / c^{2}}}-\sqrt{1-\frac{r_{0}^{2} E_{0}^{2}}{c^{2} M^{2}}}=n_{r} \hbar .(74)
$$

Introducing the dimensionless energy $E$ in accordance with (25) after cumbersome algebraic transformations the last equation is reduced to the form

$$
E=\left[1+\frac{\alpha^{\prime 2}}{\left(\sqrt{n_{\phi}{ }^{2}-\alpha^{\prime 2}}+n_{r}\right)^{2}}\right]^{-1 / 2}, \quad \alpha^{\prime}=\alpha E, \quad \alpha=\frac{e^{2}}{\hbar c},(75)
$$

where $\alpha$ is the fine structure constant. The last relation with the renormalized fine structure constant $\alpha^{\prime}$ exactly coincides with the relation of the Sommerfeld fine structure [12] obtained by means of the solution of the Dirac equation when the electron moves in the Coulomb field. As when motion of the electron in the atom the relation is fulfilled with pinpoint order of accuracy

$$
E=1-\varepsilon,(76)
$$

where $\varepsilon$ is the small positive value and the fine structure constant $\alpha=1 / 137$, then it is obviously that in our case the first approximation corresponding to zero $\varepsilon$ in the right side of equation (75) results in the exact Sommerfeld formula. 
The physical meaning of $\varepsilon$ value is the total dimensionless energy of the electron in the proton field with the reversed sign after deduction of the dimensionless rest energy. Taking into account that $\alpha^{\prime}=1$ we obtain from (75) and (76) the expression

$$
\varepsilon=\frac{\alpha^{2}}{2 n^{2}}\left[1+\frac{\alpha^{\prime 2}}{n}\left(\frac{1}{n_{\phi}}-\frac{3}{4 n}\right)\right], \quad n=n_{r}+n_{\phi}, \quad \alpha^{\prime 2}=\alpha^{2}\left(1-\frac{\alpha^{2}}{2 n^{2}}\right) .(77)
$$

Transferring to the dimension energy $W=-\varepsilon m_{0} c^{2}$ and introducing $n_{\phi}=j+1 / 2$ we obtain in the prescribed approximation the following formula for the level energy in hydrogen atom

$$
W=-\frac{\alpha^{2} m_{0} c^{2}}{2 n^{2}}-\frac{\alpha^{4} m_{0} c^{2}}{2 n^{3}}\left(\frac{1}{j+1 / 2}-\frac{3}{4 n}\right)
$$

Let us analyze the last formula. As is known from the quantum mechanics the first term determines energy levels of hydrogen atom calculated by means of the nonrelativistic Schrödinger equation. The second term represents the additive which is stipulated for the fine level splitting. This additive is calculated by means of the Dirac equation. The second term in the formula (78) in the prescribed approximation exactly coincides with the Dirac theory and the first term in (78) slightly differes from the conventional one. In our case energy levels are slightly underrated as compared with standard $W_{s}$ values. In order to compare we represent specific values

$$
\frac{W_{s}-W}{W_{s}} \cdot 100 \%=\frac{\alpha^{2}}{2 n^{2}} \cdot 100 \%=\frac{1}{n^{2}} 0.00266 \%(79)
$$

The obtained estimation is within the error estimate of theoretical and experimental energy values of the Balmer series. The total width of the fine structure [13] determined as the distance between $j_{1}=n-1 / 2$ and $j_{2}=1 / 2$ levels at prescribed $n$ coincides with the similar value from the Dirac theory. Let us consider the quantization of circular electron orbits in the proton field with the effective potential energy (32). Our aim is to ascertain how above mentioned circular orbits in the proton field (35) similar to the Caplan orbits in GRT with the Bohr orbits in hydrogen atom are connected. Obviously in the case of circular orbits we must nullify the radial quantum number $n_{r}$ in (75). Then the orbital quantum number $n_{\phi}$ will coincide with the main $n$. This results in the following value of the dimensionless energy $E$.

$$
E=\frac{1}{\sqrt{1+\frac{\alpha^{2}}{n^{2}}}}, \quad n=n_{\phi}, \quad \alpha=\frac{e^{2}}{\hbar c} .(80)
$$

It is clear that owing to smallness of the fine structure constant $\alpha$ we write the last relation in the form

$$
E=1-\frac{\alpha^{2}}{2 n^{2}}+\frac{3 \alpha^{4}}{8 n^{4}}
$$


Let us consider stable electron orbits in the proton field in accordance with the relation (35) selecting the plus sign before the radii. We consider the case when $a>>1$ that is equivalent to $r / r_{0} \gg>1$. Just that very case is realized in the hydrogen atom when radii of Bohr orbits are considerably greater than the electron classical radius. The relation (35) is transformed into the form

$$
x_{1}=a^{2}\left(2-1 / a^{2}\right), \quad E_{1}=1-\frac{1}{8 a^{2}} .
$$

Equating the energy of stable Caplan orbits from (82) to the energy of stable Bohr orbits (81), restricting by terms with $\alpha^{2}$ and using (33) we obtain the expression

$$
M=\frac{n m_{e} c r_{0}}{\alpha}=n \hbar .(83)
$$

It follows from the last relation that Caplan stable orbits in hydrogen atom exactly councide with Bohr orbits.

Let us consider the nearest to the cenrte stable Caplan orbit which in accordance with the above mentioned has the value $a^{2}=2$ or $r_{1}=4 r_{0}$. For this case we find from formulas (35)

$$
E_{1}=\sqrt{\frac{27}{32}} \cdot(84)
$$

Equating the energy from the last energy formula from the relation (80) we obtain

$$
n=\sqrt{\frac{27}{5}} \alpha=0.017 .(85)
$$

It is clear that last formula does not satisfy the quantization conditions as $n$ should be integral and positive number. It tells that the developed Sommerfeld quantization method does not work at short distances from the proton centre.

Concerning unstable Caplan orbits for the proton, none of them is not compatible with Sommerfeld quantization conditions. Thus, the applied Bohr - Sommerfeld quantization method in the Riemannian space results in the findings close to the Sommerfeld ones. It tells that the proposed version of the new metric theory at least is not absurd.

In conclusion we point out that the proposed work is the further development of a new original theory of bound charges which first was published in $[2,3,14,15]$. Electrostatic field of bound charges determines the Riemann space-time geometry non-connected with the Einstein's general relativity theory but restricting the domain of its applicability in some particular cases. This proves in [15]. Similarly to general relativity theory the elementary problems are solved at the field of bound charges and they are compared with the GRT results by means of the simplest substitution of the coupling constants. Quantization of adiabatic invariants is realized. One of the most valuable results of the theory of bound charges is the cancellation of divergence of the field of point charges [16].

\section{REFERENCES}

[1] Podosenov S. A., (1982) in col. Discussion problems of the theory of relativity and gravitation, Nauka, Moscow, pp. 95.

[2] Podosenov S. A., (2000) "The space, time and the classic fields of the bound structures," Izd. 
"Sputnik+" Company, Moscow.

[3] Podosenov S. A., Potapov A. A., Sokolov A. A., (2003) "The pulse electrodynamics of the wideband radio systems and the fields of the bound structures," "Radiotekhnika", Moscow.

[4] Podosenov S. A., (2011) "The new method of the field calculation in the space-time of the bound structures," Monograph, LAP LAMBERT Academic Publishing.

[5] Podosenov S. A., Foukzon J., Potapov A. A., (2010) "A Study of the Motion of a Relativistic Continuous Medium, Gravitation and Cosmology", Gravitation and Cosmology, Vol. 16, No 4, pp. 307-312.

[6] Podosenov S. A., Foukzon J., and Potapov A. A., (2012) "Electrodynamics of a Continuous Medium in a System with Specified Structure", Physics of Wave Phenomena, Vol. 20, No. 2, pp. 143-157.

[7] Podosenov S. A., (1997) Russian Physics Journal, Vol. 40, No. 10, pp. 985. Springer, New York, ISSN 1064-8887 (Print), 1573-9228 (Online).

[8] Landau L. D., Lifshits E. M., (1973) “Field theory,” Nauka, Moscow.

[9] Zel'dovich Ia. B., Novickov I. D., (1967) “Relativistic astrophysics,” Nauka, Moscow.

[10] Zommerfel'd A., (1958) “Electrodynamics,” IL, Moscow.

[11] Bergman P. G., (1947) “Introduction in relativity theory,” IL, Moscow.- 380 p.

[12] Berestetskiy V. B., Lifshits E. M., Pitaevsckiy L. P., (1968) "Relativistic quantum theory,” Part 1, Nauka, Moscow.- 480 p.

[13] Davidov A. S., (1963) "Quantum mechanics," Fiz-matgiz, Moscow.- 748 p.

[14] Podosenov S. A., Potapov A. A., Foukzon J. and Men'kova E. R., (2014) "Geometry of Noninertial Bases in Relativistic Mechanics Continua and Bell's Problem Solution," International Journal of Recent Advances in Physics IJRAP. Wireilla Scientific Publications, Vol. 3, No. 1, pp. 23-37.

[15] Podosenov S. A., Foukzon J., Potapov A. A., and Men'kova E. R., (2014) "About Nonlinear Classic Field Theory of Connected Charges," International Journal of Recent Advances in Physics IJRAP. Wireilla Scientific Publications, Vol. 3, No. 2, pp. 1-20.

[16] Podosenov S. A., (1997) "Space-time structure and fields of bound charges," Izvestyia Vuzov, Vol.40, No. 10, pp. 63-74.

\section{Authors}

Stanislav A. Podosenov (M'96) was born in Arkhangelsk, Russia in 1937. He received the degree in theoretical physics in 1963 and the $\mathrm{Ph}$. D. degree in relativistic mechanics of deformable media in 1972, both from Moscow State University physical Faculty, Russia. From 1963 to 1970, he lectured physics at Moscow State Technical University, where he completed his postgraduate study. Since 1970, he has been working at the All-Russian Research Institute of Optophysical Measurements on problems of relativistic mechanics and electrodynamics, noninertial frames of reference in special and general relativity, and nonstationary neutron transfer theory. Presently, he is working in the field of EM pulses radiation theory and their interaction with conducting bodies. Dr. Podosenov is the laureate of prize of the Russian Federation government 2002 in the field of science and engineering.

Jaykov Foukzon was born in 1954. He is the Doctor of physical and mathematical Sciences, Professor of Israel Institute of Technology, Haifa, Israel. The main subjects of scientific works: mathematical physics, differential geometry of manifolds, transcendental number theory, non-classical set theory, deterministic chaos and fractals, differential game theory, modern theory of homing and computer-aided design of missilery.

Alexander A. Potapov was born in 1951. He is the Doctor of physical and mathematical Sciences. Dr. Potapov has been working as a chief researcher at the Institute of radio engineering and electronics of Russian Academy of Sciences named after V. A. Kotelnikov. He is working in the field of radio physics, dispersion and diffraction of electromagnetic waves, radiolocation, recognition and image and signal processing, determinate chaos, modern topology, fractal analysis, fractional operators, scaling effects. Dr. Potapov is the academician of Russian Academy of Natural Sciences RANS and the academician of Academy of Engineering Sciences named after A. M. Prokhorov. He is the professor of Kazan State Technical University named after A. N. Tupolev. Dr. Potapov is the Chief Editor of the magazine "Nonlinear world", Member of editorial board of the international magazine "Electromagnetic Phenomena". He is Honorable radio operator of the Russian Federation. Dr. Potapov is the author of the basic researches in the field of fractal approaches to radio physics, radio engineering and radar problems 
and broad spectrum of adjacent scientific and technology paths. He is working in this field from 1979. Dr. Potapov is the initiator of the pioneer researches and developments on fractal theory application in radio physics, scaling effects and fractal operators in radio systems in Russia. He is the author of more than 400 scientific publications, includes 10 monographs.

Elena R. Men'kova was born in Moscow, Russia in 1955. She received the Ph.D. degree in 1984 from Russian Chemical-technological University, Russia. Since 1984 she has been working as a Junior Researcher, and then Senior Researcher at the All-Russian Research Institute of Optophysical Measurements. Her research interest focuses on the works connected with radiation and measurement of pulse electromagnetic fields. 